\begin{tabular}{|c|l|}
\hline Title & $\begin{array}{l}\text { A REMARK ON THE NORMA LIZA TION OF THE STA NDA RD ZETA VA LUES FOR SIEGEL MODULAR } \\
\text { FORMS }\end{array}$ \\
\hline Author(s) & Katsurada, Hidenori \\
\hline Citation & Hokkaido University Preprint Series in Mathematics, 942, 1-11 \\
\hline Issue Date & 2009-6-1 \\
\hline DOI & 10.14943/84089 \\
\hline Doc URL & http://hdl.handle.net/2115/69749 \\
\hline Type & bulletin (article) \\
\hline File Information & pre942.pdf \\
\hline
\end{tabular}

Instructions for use 


\title{
A REMARK ON THE NORMALIZATION OF THE STANDARD ZETA VALUES FOR SIEGEL MODULAR FORMS
}

\author{
HIDENORI KATSURADA
}

\begin{abstract}
In [8], we gave the normalization of the standard zeta values for Siegel modular forms, and considered the relationship between such values and congruence of cuspidal Hecke eigenforms. In this paper we give more reasonable normalization for such values and improve our previous result.
\end{abstract}

\section{INTRODUCTION}

For a cuspidal Hecke eigenform $f$ of weight $k$ with respect to $S p_{n}(\mathbf{Z})$, let $L(f, s, \underline{\mathrm{St}})$ be the standard zeta function of $f$. Then the value $\frac{L(f, m, \underline{\mathrm{St}})}{<f, f>\pi^{-n(n+1) / 2+n k+(n+1) m}}$ belongs to $\mathbf{Q}(f)$ for a certain integer $m$ if all the Fourier coefficients of $f$ belong to $\mathbf{Q}(f)$, where $\langle f, f\rangle$ is the Petersson product and $\mathbf{Q}(f)$ is the field over $\mathbf{Q}$ generated by all Hecke eigenvalues (cf. [3], [10]). In [8], to consider the relationship between these values and the congruence of Hecke eigenvalues of cusp forms, we proposed a naive way of normalizing the standard zeta values for a cuspidal Hecke eigenform $f$. This normalization is reasonable in case the multiplicity one property holds for the space of cusp forms to which $f$ belongs, and has an application to the congruence between Saito-Kurokawa lifts and non-Saito Kurokawa lifts even in case the multiplicity one property does not hold. However, this normalization is not necessarily uniquely determined by the Hecke eigenvalues of $f$. In this paper, we give a more natural way of normalization, which depends only on the system of eigenvalues.

Notation. For a commutative ring $R$, we denote by $M_{m n}(R)$ the set of $(m, n)$-matrices with entries in $R$. In particular put $M_{n}(R)=$ $M_{n n}(R)$. Here we understand $M_{m n}(R)$ the set of the empty matrix if $m=0$ or $n=0$. For an $(m, n)$-matrix $X$ and an $(m, m)$-matrix $A$, we write $A[X]={ }^{t} X A X$, where ${ }^{t} X$ denotes the transpose of $X$. Let $a$ be an element of $R$. Then for an element $X$ of $M_{m n}(R)$ we often 
use the same symbol $X$ to denote the coset $X \bmod a M_{m n}(R)$. Put $G L_{m}(R)=\left\{A \in M_{m}(R) \mid \operatorname{det} A \in R^{*}\right\}$, where $\operatorname{det} A$ denotes the determinant of a square matrix $A$, and $R^{*}$ denotes the unit group of $R$. Let $S_{n}(R)$ denote the set of symmetric matrices of degree $n$ with entries in $R$. Furthermore, for an integral domain $R$ of characteristic different from 2 , let $\mathcal{H}_{n}(R)$ denote the set of half-integral matrices of degree $n$ over $R$, that is, $\mathcal{H}_{n}(R)$ is the set of symmetric matrices of degree $n$ whose $(i, j)$-component belongs to $R$ or $\frac{1}{2} R$ according as $i=j$ or not. For a subset $S$ of $M_{n}(R)$ we denote by $S^{\times}$the subset of $S$ consisting of non-degenerate matrices. In particular, if $S$ is a subset of $S_{n}(\mathbf{R})$ with $\mathbf{R}$ the field of real numbers, we denote by $S_{>0}$ (resp. $S_{\geq 0}$ ) the subset of $S$ consisting of positive definite (resp. semi-positive definite) matrices. Let $R^{\prime}$ be a subring of $R$. Two symmetric matrices $A$ and $A^{\prime}$ with entries in $R$ are called equivalent over $R^{\prime}$ with each other and write $A \widetilde{R^{\prime}} A^{\prime}$ if there is an element $X$ of $G L_{n}\left(R^{\prime}\right)$ such that $A^{\prime}=A[X]$. We also write $A \sim A^{\prime}$ if there is no fear of confusion. For square matrices $X$ and $Y$ we write $X \perp Y=\left(\begin{array}{cc}X & O \\ O & Y\end{array}\right)$.

\section{Siegel-Eisenstein SERIES AND Differential Operators}

For a complex number $x$ put $\mathbf{e}(x)=\exp (2 \pi \sqrt{-1} x)$. Furthermore put $J_{n}=\left(\begin{array}{cc}O_{n} & -1_{n} \\ 1_{n} & O_{n}\end{array}\right)$, where $1_{n}$ denotes the unit matrix of degree $n$. For a subring $K$ of $\mathbf{R}$ put

$G S p_{n}(K)^{+}=\left\{M \in G L_{2 n}(K) \mid J_{n}[M]=\kappa(M) J_{n}\right.$ with some $\left.\kappa(M)>0\right\}$, and

$$
S p_{n}(K)=\left\{M \in G S p_{n}(K)^{+} \mid J_{n}[M]=J_{n}\right\} .
$$

Furthermore, put

$$
\Gamma^{(n)}=S p_{n}(\mathbf{Z})=\left\{M \in G L_{2 n}(\mathbf{Z}) \mid J_{n}[M]=J_{n}\right\} .
$$

Let $\mathbf{H}_{n}$ be Siegel's upper half-space. For each element $M=\left(\begin{array}{cc}A & B \\ C & D\end{array}\right) \in$ $G S p_{n}(\mathbf{R})^{+}$and $Z \in \mathbf{H}_{n}$ put

$$
M(Z)=(A Z+B)(C Z+D)^{-1}
$$

and

$$
j(M, Z)=\operatorname{det}(C Z+D) .
$$

Furthermore, for a function $f$ on $\mathbf{H}_{n}$ we define $\left.f\right|_{k} M$ as

$$
\left(\left.f\right|_{k} M\right)(Z)=\operatorname{det}(M)^{k / 2} j(M, Z)^{-k} f(M(Z)) .
$$


A function $f$ on $\mathbf{H}_{n}$ is called a $C^{\infty}$-modular form of weight $k$ with respect to $\Gamma^{(n)}$ if it satisfies the following conditions:

(i) $f$ is a $C^{\infty}$-function on $\mathbf{H}_{n}$;

(ii) $\left(\left.f\right|_{k} M\right)(Z)=f(Z)$ for any $M \in \Gamma^{(n)}$.

We call a $C^{\infty}$-modular form $f$ a holomorphic modular form if (i) $f$ is holomorphic on $\mathbf{H}_{n}$;

(ii) if $n=1, f(z)$ is bounded on the set $\{x+\sqrt{-1} y \mid y \geq \alpha\}$ for each $\alpha>0$.

We denote by $\mathfrak{M}_{k}\left(\Gamma^{(n)}\right)$ (resp. $\mathfrak{N}_{k}^{\infty}\left(\Gamma^{(n)}\right)$ the space of holomorphic (resp. $C^{\infty}{ }_{-}$) modular forms of weight $k$ with respect to $\Gamma^{(n)}$. For a holomorphic modular form $f$ of weight $k$ with respect to $\Gamma^{(n)}$, let

$$
f(Z)=\sum_{A \in \mathcal{H}_{n}(\mathbf{Z})_{\geq 0}} a_{f}(A) \mathbf{e}(\operatorname{tr}(A Z)),
$$

be the Fourier expansion of $f(Z)$, where tr denotes the trace of a matrix. We call $f(Z)$ a cusp form if $a_{f}(A)=0$ unless $A$ is positive-definite. We denote by $\mathfrak{\Xi}_{k}\left(\Gamma^{(n)}\right)$ the submodule of $\mathfrak{R}_{k}\left(\Gamma^{(n)}\right)$ consisting of cusp forms. Let $d v$ denote the invariant volume element on $\mathbf{H}_{n}$ defined by $d v=\operatorname{det}(\operatorname{Im}(Z))^{-n-1} \wedge_{1 \leq j \leq l \leq n}\left(d x_{j l} \wedge d y_{j l}\right)$. Here for $Z \in \mathbf{H}_{n}$ we write $Z=\left(x_{j l}\right)+\sqrt{-1}\left(y_{j l}\right)$ with real matrices $\left(x_{j l}\right)$ and $\left(y_{j l}\right)$. For two $C^{\infty}$ modular forms $f$ and $g$ of weight $k$ with respect to $\Gamma^{(n)}$ we define the Petersson scalar product $<f, g\rangle$ by

$$
<f, g>=\int_{\Gamma^{(n)} \backslash \mathbf{H}_{n}} f(Z) \overline{g(Z)} \operatorname{det}(\operatorname{Im}(Z))^{k} d v,
$$

provided the integral converges.

For a positive even integer $k$ we define the Siegel Eisenstein series $E_{n, k}(Z, s)$ of degree $n$ as

$E_{n, k}(Z, s)=\zeta(1-k-2 s) \prod_{i=1}^{[n / 2]} \zeta(1-2 k-4 s+2 i) \sum_{M \in \Gamma_{\infty}^{(n)} \backslash \Gamma^{(n)}} j(M, Z)^{-k}(\operatorname{det}(\operatorname{Im}(M(Z))))^{s}$

$\left(Z \in \mathbf{H}_{n}, s \in \mathbf{C}\right.$ ), where $\zeta(*)$ is Riemann's zeta function, and $\Gamma_{\infty}^{(n)}=$ $\left\{\left(\begin{array}{cc}* & * \\ O_{n} & *\end{array}\right) \in \Gamma^{(n)}\right\}$. Then $E_{n, k}(Z, s)$ is holomorphic at $s=0$ as a function of $s$. Furthermore, assume that $k \geq(n+1) / 2$. Then $E_{n, k}(Z, 0)$ is holomorphic as a function of $Z$ unless $k=(n+2) / 2 \equiv 2 \bmod 4$, or $k=(n+3) / 2 \equiv 2 \bmod 4$ (cf. [11]). From now on we assume 
that $E_{n, k}(Z, 0)$ is holomorphic as a function of $Z$, and write $E_{n, k}(Z)=$ $E_{n, k}(Z, 0)$.

Now we review the differential operators acting on the space of modular forms following [5], [7]. Let $X=\left(x_{i j}\right)_{1 \leq i \leq m, 1 \leq j \leq d}$ be a matrix of variables, and for $1 \leq i, j \leq m$, put $\Delta_{i, j}=\sum_{\nu=1}^{d} \frac{\partial^{2}}{\partial x_{i \nu} \partial x_{j \nu}}$. A polynomial $P(X)$ in $X$ is called pluriharmonic if $\Delta_{i j} P=0$ for any $1 \leq i, j, \leq m$. Take a polynomial mapping $P\left(X_{1}, X_{2}\right)$ from $M_{n, 2 l}(\mathbf{C}) \times M_{n, 2 l}(\mathbf{C})$ to $\mathbf{C}$ such that

D-1. $P\left(X_{1}, X_{2}\right)$ is pluriharmonic for each $X_{i}(i=1,2)$.

D-2. $P\left(X_{1} g, X_{2} g\right)=P\left(X_{1}, X_{2}\right)$ for any $g \in O(2 l)$, where $O(2 l)$ is the orthogonal group of degree $2 l$.

D-3. $P\left(a_{1} X_{1}, a_{2} X_{2}\right)=\left(\operatorname{det} a_{1}\right)^{\nu}\left(\operatorname{det} a_{2}\right)^{\nu} P\left(X_{1}, X_{2}\right)$ for $a_{1}, a_{2} \in G L_{n}(\mathbf{C})$. Assume that $l \geq n$. Then there exists a unique polynomial mapping $Q(W)$ from $S_{2 n}(\mathbf{C})$ to $\mathbf{C}$ s.t $P\left(X_{1}, X_{2}\right)=Q\left(\left(\begin{array}{cc}X_{1}{ }^{t} X_{1} & X_{1}{ }^{t} X_{2} \\ X_{2}{ }^{t} X_{1} & X_{2}{ }^{t} X_{2}\end{array}\right)\right)$. We note that $\operatorname{deg} Q=n \nu$. Let $Z=\left(z_{i j}\right)_{1 \leq i, j \leq 2 n}$ be a matrix of variables with $z_{i j}=z_{j i}$, and we write $\frac{\tilde{\partial}}{\partial z_{i j}}=\frac{\left(1+\delta_{i j}\right)}{2} \frac{\partial}{\partial z_{i j}}$, and $\left(\frac{\partial}{\partial Z}\right)=\left(\frac{\tilde{\partial}}{\partial z_{i j}}\right)_{1 \leq i, j \leq 2 n}$. For $f \in C^{\infty}\left(\mathbf{H}_{2 n}\right)$ we define $\mathcal{D}_{Q}(f)$ and $\tilde{\mathcal{D}}_{Q}(f)$ by

$$
\mathcal{D}_{Q}(f)=Q\left(\frac{\partial}{\partial Z}\right)(f)
$$

and

$$
\tilde{\mathcal{D}}_{Q}(f)=\mathcal{D}_{Q}(f)_{Z_{12}=0},
$$

where we write $Z=\left(\begin{array}{cc}Z_{1} & Z_{12} \\ { }^{t} Z_{12} & Z_{2}\end{array}\right)$ with $Z_{1}, Z_{2} \in \mathbf{H}_{n}$ and $Z_{12} \in$ $M_{n}(\mathbf{C})$.

For a subspace $\mathfrak{M}$ of $\mathfrak{M}_{l}^{\infty}\left(\Gamma^{(n)}\right)$ let

$$
\mathfrak{R} \otimes \mathfrak{R}=\left\{\sum_{i, j} a_{i j} f_{i}\left(Z_{1}\right) f_{j}\left(Z_{2}\right) \text { (finite sum); } f_{i}, f_{j} \in \mathfrak{R}, a_{i j} \in \mathbf{C}\right\} .
$$

Put $C_{q}(s)=s(s+1 / 2) \cdots(s+(q-1) / 2)$. We choose $Q=Q_{n, l}^{\nu}$ such that

$$
\tilde{\mathcal{D}}_{Q_{n, l}^{\nu}}\left(\operatorname{det} Z_{12}^{\nu}\right)=(-1)^{n \nu} \prod_{\mu=1}^{\nu}\left(C_{n}(\mu / 2) C_{n}(l-n+\nu-\mu / 2)\right),
$$

and put

$$
\stackrel{\circ}{\mathcal{D}}_{n, l}^{\nu}=\tilde{\mathcal{D}}_{Q_{n, l}^{\nu}} \text {. }
$$

This coincides with $\stackrel{\circ}{\mathfrak{D}}_{n, l}^{\nu}$ in [5]. Let $n$ and $l$ be integers. Assume that $l \geq n+3$ or $l \geq n+1$ according as $n \equiv 1 \bmod 4$ or not. Then $E_{2 n}(Z, 0)$ 
is holomorphic modular form and write $E_{2 n, l}(Z)=E_{2 n, l}(Z, 0)$ as above. We then define $\mathcal{E}_{2 n, l, k}\left(z_{1}, z_{2}\right)$ as

$$
\mathcal{E}_{2 n, l, k}\left(z_{1}, z_{2}\right)=(-1)^{l / 2+1} 2^{-n}(2 \pi \sqrt{-1})^{(l-k) n}(l-n) \stackrel{\circ}{\mathcal{D}}_{n, l}^{k-l}\left(E_{2 n, l}\right)\left(z_{1}, z_{2}\right),
$$

where $z_{1}, z_{2} \in \mathbf{H}_{n}$. Then we see the following (cf. Section 4 of [8].)

Theorem 2. In addition to the notation and the assumption as above, assume that $k>l$. Then $\mathcal{E}_{2 n, l, k}\left(z_{1}, z_{2}\right)$ belongs to $\mathfrak{S}_{k}\left(\Gamma^{(n)}\right) \otimes$ $\mathfrak{\Xi}_{k}\left(\Gamma^{(n)}\right)$. Furthermore, all the Fourier coefficients of $\mathcal{E}_{2 n, l, k}\left(z_{1}, z_{2}\right)$ are rational and any prime divisor of its denominator is not greater than $(2 k-1) !$

\section{Pullback formula}

Let $\mathbf{L}_{n}=\mathbf{L}_{\mathbf{Q}}\left(G S p_{n}(\mathbf{Q})^{+}, \Gamma^{(n)}\right)$ denote the Hecke ring over $\mathbf{Q}$ associated with the Hecke pair $\left(G S p_{n}(\mathbf{Q})^{+}, \Gamma^{(n)}\right)$. For a prime number $p$ put

$$
T(p)=\Gamma^{(n)}\left(1_{n} \perp p 1_{n}\right) \Gamma^{(n)},
$$

and

$$
\left(p^{ \pm}\right)=\Gamma^{(n)}\left(p^{ \pm} 1_{2 n}\right) \Gamma^{(n)} .
$$

Furthermore, for $i=1, \ldots, n-1$ put

$$
T_{i}\left(p^{2}\right)=\Gamma^{(n)}\left(1_{n-i} \perp p 1_{i} \perp p^{2} 1_{n-i} \perp p 1_{i}\right) \Gamma^{(n)} .
$$

As is well known, $\mathbf{L}_{n}$ is generated over $\mathbf{Q}$ by all $T(p),\left(p^{ \pm}\right)$and $T_{i}\left(p^{2}\right)(i=$ $1, \ldots, n-1)$. We denote by $\mathbf{L}_{n}^{\prime}$ the subalgebra of $\mathbf{L}_{n}$ generated by over $\mathbf{Z}$ by all $T(p)$ and $T_{i}\left(p^{2}\right)(i=1, \ldots, n)$. Let $T=\Gamma^{(n)} M \Gamma^{(n)}$ be an element of $\mathbf{L}_{n} \otimes \mathbf{C}$. Write $T$ as $T=\cup_{\gamma} \Gamma^{(n)} \gamma$ and for $f \in \mathfrak{M}_{k}\left(\Gamma^{(n)}\right)$ define the Hecke operator $\left.\right|_{k} T$ associated to $T$ as

$$
\left.f\right|_{k} T=\left.\operatorname{det}(M)^{k / 2-(n+1) / 2} \sum_{\gamma} f\right|_{k} \gamma .
$$

We call this action the Hecke operator as usual (cf. [1].) If $f$ is an eigenfunction of a Hecke operator $T \in \mathbf{L}_{n} \otimes \mathbf{C}$, we denote by $\lambda_{f}(T)$ its eigenvalue. We call $f \in \mathfrak{M}_{k}\left(\Gamma^{(n)}\right)$ a Hecke eigenform if it is a common eigenfunction of all Hecke operators. Furthermore, we denote by $\mathbf{Q}(f)$ the field generated over $\mathbf{Q}$ by eigenvalues of all $T \in \mathbf{L}_{n}$ as in Section 1. As is well known, $\mathbf{Q}(f)$ is a totally real algebraic number field of finite degree. Now, first we consider the integrality of the eigenvalues of Hecke operators. For an algebraic number field $K$, let $\mathfrak{D}_{K}$ denote the ring of integers in $K$. The following result is due to Mizumoto [9] (see also [8].) 
Theorem 3.1. Let $k \geq n+1$. Let $f \in \mathfrak{\Xi}_{k}\left(\Gamma^{(n)}\right)$ be a Hecke eigenform. Then $\lambda_{f}(T)$ belongs to $\mathfrak{D}_{\mathbf{Q}(f)}$ for any $T \in \mathbf{L}_{n}^{\prime}$.

Put $G S p_{n}\left(\mathbf{Q}_{p}\right)=\left\{M \in G L_{2 n}\left(\mathbf{Q}_{p}\right) ; J_{n}[M]=\kappa(M) J_{n}\right.$ with some $\left.\kappa(M) \in \mathbf{Q}_{p}^{\times}\right\}$, and let $\mathbf{L}_{n p}=\mathbf{L}\left(G S p_{n}\left(\mathbf{Q}_{p}\right), G S p_{n}\left(\mathbf{Q}_{p}\right) \cap G L_{2 n}\left(\mathbf{Z}_{p}\right)\right)$ be the Hecke algebra associated with the pair $\left(G S p_{n}\left(\mathbf{Q}_{p}\right), G S p_{n}\left(\mathbf{Q}_{p}\right) \cap G L_{2 n}\left(\mathbf{Z}_{p}\right)\right)$. Now assume that $f$ is a common eigenfunction of all Hecke operators, and for each prime number $p$, let $\alpha_{0}(p), \alpha_{1}(p), \ldots, \alpha_{n}(p)$ be the Satake parameters of $\mathbf{L}_{n p}$ determined by $f$. We then define the standard zeta function $L(f, s, \underline{\mathrm{St}})$ by

$$
L(f, s, \underline{\mathrm{St}})=\prod_{p} \prod_{i=1}^{n}\left\{\left(1-p^{-s}\right)\left(1-\alpha_{i}(p) p^{-s}\right)\left(1-\alpha_{i}(p)^{-1} p^{-s}\right)\right\}^{-1}
$$

Let $f(z)=\sum_{A \in \mathcal{H}_{n}(\mathbf{Z})_{>0}} a(A) \mathbf{e}(\operatorname{tr}(A z))$ be a Hecke eigenform in $\mathfrak{\Xi}_{k}\left(\Gamma^{(n)}\right)$. For a positive integer $m \leq k-n$ such that $m \equiv n \bmod 2$ put

$$
\begin{aligned}
& \tilde{\Lambda}(f, m, \underline{\mathrm{St}})=(-1)^{n(-m+1) / 2+1} 2^{-4 k n+3 n^{2}+n+(n-1) m+2} \\
& \times \Gamma(m+1) \prod_{i=1}^{n} \Gamma(2 k-n-i) \frac{L(f, m, \underline{\mathrm{St}})}{\pi^{-n(n+1) / 2+n k+(n+1) m}},
\end{aligned}
$$

and

$$
\Lambda(f, m, \underline{\mathrm{St}})=\frac{\tilde{\Lambda}(f, m, \underline{\mathrm{St}})}{<f, f>} .
$$

Then the following result is due to [3] and [10]:

Theorem 3.2. Let $l, k$ and $n$ be a positive integers. Assume that $k$ and $l+n$ are even, and $3 \leq l \leq k-n$ or $1 \leq l \leq k-n$ according as $n \equiv 1 \bmod 4$ or not. Let $f \in \mathfrak{S}_{k}\left(\Gamma^{(n)}\right)$ be a Hecke eigenform. Then we have

$$
\left.<f, \mathcal{E}_{2 n, l+n, k}(*,-\bar{z})\right)>=\Lambda(f, l, \underline{\mathrm{St}}) f(z) .
$$

Now assume that $l<k$. Then we have the following Fourier expansion of $\mathcal{E}_{2 n, l, k}\left(z_{1}, z_{2}\right)$ as a function of $z_{1}$ :

$$
\mathcal{E}_{2 n, l, k}\left(z_{1}, z_{2}\right)=\sum_{A_{1} \in \mathcal{H}_{n}(\mathbf{Z})_{>0}} \mathcal{F}_{l, k ; A_{1}}\left(z_{2}\right) \mathbf{e}\left(\operatorname{tr}\left(A_{1} z_{1}\right)\right) .
$$


Then $\mathcal{F}_{l, k ; A_{1}}\left(z_{2}\right)$ belongs to $\mathfrak{\Xi}_{k}\left(\Gamma^{(n)}\right)$ Take an orthogonal basis $\left\{f_{i}\right\}_{i=1}^{d_{1}}$ of $\mathfrak{\Xi}_{k}\left(\Gamma^{(n)}\right)$ consisting of Hecke eigenforms. Write

$$
f_{i}(z)=\sum_{A \in \mathcal{H}_{n}(\mathbf{Z})_{>0}} a_{i}(A) \mathbf{e}(\operatorname{tr}(A z)) .
$$

Now we have the following formula.

Theorem 3.3. (Theorem 4.4 of [8]) In addition to the above notation and the assumption, assume that $l \leq k-n-2$. Then for any positive definite half-integral matrix $A_{1}$ of degree $n$ we have

$$
\mathcal{F}_{l+n, k ; A_{1}}(z)=\sum_{i=1}^{d_{1}} \Lambda\left(f_{i}, l, \underline{\mathrm{St}}\right) a_{i}\left(A_{1}\right) \overline{f_{i}(-\bar{z})} .
$$

\section{Congruence of modular forms}

In this section we consider the congruence between the Hecke eigenvalues of modular forms of the same weight. Let $K$ be an algebraic number filed, and $\mathfrak{D}=\mathfrak{D}_{K}$ the ring of integers in $K$. For a prime ideal $\mathfrak{P}$ of $\mathfrak{D}$, we denote by $\mathfrak{D}_{(\mathfrak{P})}$ the localization of $\mathfrak{D}$ at $\mathfrak{P}$ in $K$. Let $\mathfrak{A}$ be a fractional ideal in $K$. If $\mathfrak{A}=\mathfrak{P}^{e} \mathfrak{B}$ with $\mathfrak{B D}_{(\mathfrak{P})}=\mathfrak{D}_{(\mathfrak{P})}$ we write $\operatorname{ord}_{\mathfrak{P}}=e$. We simply write $\operatorname{ord}_{\mathfrak{P}}(c)=\operatorname{ord}_{\mathfrak{P}}((c))$ for $c \in K$. For a Q-algebra homomorphism $\lambda: \mathbf{L}_{n} \longrightarrow \mathbf{C}$ let

$$
\mathfrak{\Im}_{k}\left(\Gamma^{(n)}, \lambda\right)=\left\{f \in \mathfrak{\subseteq}_{k}\left(\Gamma^{(n)}|f| T=\lambda(T) f \text { for all } T \in \mathbf{L}_{n}\right\},\right.
$$

and $\mathbf{Q}(\lambda)$ the field over $\mathbf{Q}$ generated by all the $\lambda(T)$ 's. For a Hecke eigenform $f$, the mapping $\mathbf{L}_{n} \in T \mapsto \lambda_{f}(T) \in \mathbf{C}$ defines a Q-algebra homomorphism from $\mathbf{L}_{n}$ to $\mathbf{C}$, which is denoted by $\lambda_{f}$. Then $f$ belongs to $\mathfrak{S}_{k}\left(\Gamma^{(n)}, \lambda\right)$, and $\mathbf{Q}(\lambda)=\mathbf{Q}(f)$. Let

$$
\hat{T}_{n, k}=\left\{\lambda_{f} \mid f \text { is a Hecke eigenform in } \mathfrak{\bigotimes}_{k}\left(\Gamma^{(n)}\right)\right\} .
$$

Let $R$ be a subring of $\mathbf{C}$. Let $\mathfrak{\Xi}_{k}\left(\Gamma^{(n)}\right)(R)$ be the $\mathbf{Z}$-module consisting of elements of $\mathfrak{\Xi}_{k}\left(\Gamma^{(n)}\right)$ whose Fourier coefficients belong to $R$.

Lemma 4.1. Let $\lambda_{1}, \ldots, \lambda_{r}$ be elements of $\hat{T}_{n, k}$. For each $j$ let $f_{i 1}, \ldots, f_{i, l_{i}}$ be an elements of $\mathfrak{\Xi}_{k}\left(\Gamma^{(n)} ; \lambda_{i}\right)$ linearly independent over $\mathbf{C}$, and $G$ an element of $\mathfrak{\Xi}_{k}\left(\Gamma^{(n)}\right)$. Write

$$
f_{i j}(z)=\sum_{A} a_{i j}(A) \mathbf{e}(\operatorname{tr}(A z))
$$


for $i=1, \ldots, r, j=1, \ldots, l_{i}$, and

$$
G(z)=\sum_{A} a_{G}(A) \mathbf{e}(\operatorname{tr}(A z))
$$

Let $K$ be the composite field of $\mathbf{Q}\left(\lambda_{1}\right), \mathbf{Q}\left(\lambda_{2}\right), \ldots$, and $\mathbf{Q}\left(\lambda_{r}\right)$, and $\mathfrak{D}=$ $\mathfrak{D}_{K}$. Let $\mathfrak{P}$ be a prime ideal of $\mathfrak{D}$. Furthermore assume that

(1) $a_{G}(A)$ belongs to $\mathfrak{D}_{(\mathfrak{P})}$ for any $A \in \mathcal{H}_{n}(\mathbf{Z})_{>0}$ (2) there exist $c_{i j} \in$ $K\left(i=1, \ldots, r, j=1, \ldots, l_{i}\right)$ and $A \in \mathcal{H}_{n,>0}$ such that $\operatorname{ord}_{\mathfrak{P}}\left(\sum_{j=1}^{l_{1}} c_{1 j} a_{1 j}(A)\right)<$ 0 and

$$
G(z)=\sum_{i=1}^{r} \sum_{j=1}^{l_{i}} c_{i j} f_{i j}(z) .
$$

Then there exists an $i \neq 1$ such that we have

$$
\lambda_{i}(T) \equiv \lambda_{1}(T) \bmod \mathfrak{P}
$$

for any $T \in \mathbf{L}_{n}^{\prime}$.

Proof. The assertion can be proved similarly to Lemma 5.1 of [8].

Lemma 4.2. Let $V$ be a vector space over $\mathbf{C}$ and $(*, *)$ be an Hermitian inner product on $V$. Let $\alpha$ and $\beta$ be $\mathbf{C}$-linear mappings from $V$ to C. Take an orthogonal basis $\left\{v_{i}\right\}_{i=1}^{d}$ of $V$ and put

$$
I=\sum_{i=1}^{d} \frac{\alpha\left(v_{i}\right) \overline{\beta\left(v_{i}\right)}}{\left(v_{i}, v_{i}\right)} .
$$

Then I does not depend on the choice of $\left\{v_{i}\right\}_{i=1}^{d}$.

Proof. This may be well-known or can be easily proved. But for the readers' convenience, we here give a proof. Let $\left\{u_{i}\right\}_{i=1}^{d}$ be another orthogonal basis. Then we have

$$
u_{i}=\sum_{j=1}^{d} a_{i j} v_{j}
$$

with $a_{i j} \in \mathbf{C}(i, j=1, \ldots, d)$. Put $\tilde{a}_{i j}=\frac{a_{i j} \sqrt{\left(v_{j}, v_{j}\right)}}{\sqrt{\left(u_{i}, u_{1}\right)}}$, and $\tilde{A}=\left(\tilde{a}_{i j}\right)_{d \times d}$. Then we have

$$
\frac{u_{i}}{\sqrt{\left(u_{i}, u_{1}\right)}}=\sum_{j=1}^{d} \tilde{a}_{i j} \frac{v_{j}}{\sqrt{\left(v_{j}, v_{j}\right)}}
$$


and $\left\{\frac{u_{i}}{\sqrt{\left(u_{i}, u_{1}\right)}}\right\}_{i=1}^{d}$ and $\left\{\frac{v_{i}}{\sqrt{\left(v_{i}, v_{1}\right)}}\right\}_{i=1}^{d}$ are orthonormal bases of $V$. Thus $\tilde{A}$ is an unitary matrix, and therefore we have

$$
\sum_{i=1}^{d} \frac{a_{i l} \overline{a_{i m}}}{\left(u_{i}, u_{i}\right)}=\frac{\delta_{l m}}{\left(v_{l}, v_{l}\right)},
$$

where $\delta_{l m}$ is Kronecker delta. Thus we have

$$
\begin{aligned}
& \sum_{i=1}^{d} \frac{\alpha\left(u_{i}\right) \overline{\beta\left(u_{i}\right)}}{\left(u_{i}, u_{i}\right)}=\sum_{i=1}^{d} \sum_{l, m=1}^{d} \frac{a_{i l} \overline{a_{i m}} \alpha\left(u_{l}\right) \overline{\beta\left(u_{m}\right)}}{\left(u_{i}, u_{i}\right)} \\
= & \sum_{l, m=1}^{d} \alpha\left(u_{l}\right) \overline{\beta\left(u_{m}\right)} \sum_{i=1}^{d} \frac{a_{i l} \overline{a_{i m}}}{\left(u_{i}, u_{i}\right)}=\sum_{l=1}^{d} \frac{\alpha\left(u_{l}\right) \overline{\beta\left(u_{l}\right)}}{\left(v_{l}, v_{l}\right)} .
\end{aligned}
$$

This proves the assertion.

Let $f$ be a Hecke eigenform in $\mathfrak{\subseteq}_{k}\left(\Gamma^{(n)}\right)$ and $M$ be a subspace of $\mathfrak{\Im}_{k}\left(\Gamma^{(n)}\right)$ stable under all the Hecke operators $T \in \mathbf{L}_{n}$. Assume that $M$ is contained in $(\mathbf{C} f)^{\perp}$, where $(\mathbf{C} f)^{\perp}$ is the orthogonal complement of C $f$ in $\mathfrak{\Xi}_{k}\left(\Gamma^{(n)}\right)$ with respect to the Petersson product. Let $K$ be a an algebraic number field of finite degree containing $\mathbf{Q}(f)$. A prime ideal $\mathfrak{P}$ of $\mathfrak{D}_{K}$ is called a congruence prime of $f$ with respect to $M$ if there exists a Hecke eigenform $g \in M$ such that

$$
\lambda_{f}(T) \equiv \lambda_{g}(T) \bmod \tilde{\mathfrak{P}}
$$

for any $T \in \mathbf{L}_{n}^{\prime}$, where $\tilde{\mathfrak{P}}$ is some prime ideal of $\mathfrak{D}_{K \mathbf{Q}(g)}$ lying above $\mathfrak{P}$. If $M=(\mathbf{C} f)^{\perp}$, we simply call $\mathfrak{P}$ a congruence prime of $f$.

Now we consider the relation between the congruence primes and the standard zeta values. To consider this, we have to normalize the standard zeta value $\Lambda(f, l, \underline{\mathrm{St}})$ for a Hecke eigenform $f$ because it is not uniquely determined by the system of Hecke eigenvalues of $f$. We note that there is no reasonable normalization of cuspidal Hecke eigenform in the higher degree case unlike the elliptic modular case. Thus we define the following normalization. Take an orthogonal basis $\left\{f_{i}\right\}_{i=1}^{d}$ of $\mathfrak{\Im}_{k}\left(\Gamma^{(n)}, \lambda\right)$ Let

$$
f_{i}(z)=\sum_{A} a_{i}(A) \mathbf{e}(\operatorname{tr}(A z))
$$

be the Fourier expansion of $f_{i}$. For $A$ and $B$ and an integer $l$ put

$$
I_{A, B}(l)=\sum_{i=1}^{d} \frac{a_{i}(A) \overline{a_{i}(B)} \tilde{\Lambda}\left(f_{i}, l, \underline{\mathrm{St}}\right)}{\left\langle f_{i}, f_{i}\right\rangle} .
$$


Then, we have $\tilde{\Lambda}\left(f_{i}, l, \underline{\mathrm{St}}\right)=\tilde{\Lambda}(f, l, \underline{\mathrm{St}})$ for any $i$, and by Lemma 4.2 , $I_{A, B}(l)$ does not depend on the choice of $\left\{f_{i}\right\}$. Furthermore, by Theorem of [10], we can take $\left\{f_{i}\right\}$ so that $f_{i} \in \mathbb{\Theta}_{k}\left(\Gamma^{(n)}\right)(\mathbf{Q}(f))$. Thus $\frac{\tilde{\Lambda}\left(f_{i}, l, \underline{\mathrm{St}}\right)}{\left\langle f_{i}, f_{i}\right\rangle}$ lies in $\mathbf{Q}(f)$ for any $i$. Thus $I_{A, B}(l)$ lies in $\mathbf{Q}(f)$. Let $\mathfrak{I}$ be the $\mathfrak{D}_{\mathbf{Q}(f)^{-}}$ module generated by all $I_{A, B}(l)$ 's. Then $\mathfrak{I}$ becomes a fractional ideal in $\mathbf{Q}(f)$. We note that this fractional ideal does not depend on the choice of an orthogonal basis stated above, and is uniquely determined by $l$ and $\lambda$, which will be denoted by $\mathfrak{I}(\lambda ; l)$. In particular, if we have $\operatorname{dim} \mathfrak{\Im}_{k}\left(\Gamma^{(n)}, \lambda\right)=1$, this coincides with the ideal $\Lambda(f, l, \underline{\mathrm{St}}) \mathfrak{I}_{f}^{2}$ defined in [8]. Now for a Hecke eigenform $f$ in $\mathfrak{\Xi}_{k}\left(\Gamma^{(n)}\right)$, let $\mathfrak{I}_{f}$ denote the subspace of $\mathfrak{\Xi}_{k}\left(\Gamma^{(n)}\right)$ spanned by all Hecke eigenforms with the same system of the Hecke eigenvalues as $f$. Let $K_{0}$ be the composite field of all the Hecke fields of Hecke eigenforms in $\mathfrak{\Im}_{k}\left(\Gamma^{(n)}\right)$. As stated above we can take an orthogonal basis of $\mathfrak{\Xi}_{k}\left(\Gamma^{(n)}\right)$ consisting of Hecke eigenforms whose Fourier coefficients lie in $K_{0}$. Thus by Theorem 3.3 and Lemma 4.1, we have

Theorem 4.3. Let $\lambda$ be an element of $\hat{T}_{n, k}$, and $f$ an element of $\mathfrak{\Im}_{k}\left(\Gamma^{(n)} ; \lambda\right)$. Let $l$ be a positive integer satisfying the condition in Theorem 3.2. Let $\mathfrak{P}$ be a prime ideal of $\mathfrak{D}_{K_{0}}$. Assume that $\operatorname{ord}_{\mathfrak{N}}(\mathfrak{I}(\lambda ; l))<0$ and that it does not divide $(2 k-1)$ !. Then $\mathfrak{P}$ is a congruence prime of $f$ with respect to $\mathfrak{\Im}_{k}\left(\Gamma^{(n)} ; \lambda\right)^{\perp}$. In particular, if a rational prime number $p$ divides the denominator of $N_{K_{0}}(\mathfrak{I}(\lambda ; l))$, then $p$ is divided by some congruence prime of $f$ with respect to $\mathfrak{S}_{k}\left(\Gamma^{(n)} ; \lambda\right)^{\perp}$.

Remark. In line 5, page 108 of [8], we stated that

"Then, by multiplying a suitable constant $c$ we may assume all the Fourier coefficients $a_{f}(A)^{\prime}$ are elements of $\mathbf{Q}(f)$ with bounded denominator."

This is true if the multiplicity one property in the space of cusp forms to which $f$ belongs, but it is not true in general. Thus this sentence should read:

"Then, we assume all the Fourier coefficients $a_{f}(A)^{\prime}$ are elements of $\mathbf{Q}(f)$ with bounded denominator if we multiply $f$ by a suitable constant c."

Accordingly, the first sentence of Theorem 5.2 should read:

"Let $f$ be a Hecke eigenform in $\mathfrak{\Xi}_{k}\left(\Gamma^{(n)}\right)$ satisfying the above assumption."

By Theorem of Mizumoto [10], we can take an orthogonal basis $\left\{f_{i}\right\}$ such that each $f_{i}$ is a Hecke eigenform whose Fourier coefficients lie 
in $\mathbf{Q}\left(f_{i}\right)$ in Theorem 4.4. Thus Theorem 5.2 remains valid under this assumption.

Remark. If $\hat{f}$ is the Saito-Kurokawa lift, $\hat{f}$ satisfies the above assumption. Thus Theorem 6.1 in [8] remains valid.

\section{REFERENCES}

[1] A. N. Andrianov, Quadratic forms and Hecke operators, Springer, 1987.

[2] S. Böcherer, Über die Fourier-Jacobi-Entwicklung Siegelscher Eisensteinreihen, Math. Z. 183(1983), 21-46.

[3] S. Böcherer, Über die Fourier-Jacobi-Entwicklung Siegelscher Eisensteinreihen II, Math. Z. 189(1985), 81-110.

[4] S. Böcherer, Über die Fourierkoeffizienten Siegelscher Eisensteinreihen, Manuscripta Math., 45(1984), 273-288.

[5] S. Böcherer and C.G. Schmidt, p-adic measures attached to Siegel modular forms, Ann. Inst. Fourier, 50(2000), 1375-1443.

[6] P. Garrett, Pullbacks of Eisenstein series, Automorphic forms of several variables, Progress in Math. 46, Birkhäuser, 1984, 114-137.

[7] T. Ibukiyama, On Differential operators on automorphic forms and invariant pluri-harmonic polynomials, Comm. Math. Univ. St. Pauli, 48(1999), 103-118.

[8] H. Katsurada, Congruence of Siegel modular forms and special values of their zeta functions, Math. Z. 259 (2008), 97-111

[9] S. Mizumoto, On integrality of Eisenstein liftings, Manuscripta Math. 89(1996), 203-235. Corrections Ibid.307(1997), 169-171.

[10] S. Mizumoto, Poles and residues of standard L-functions attached to Siegel modular forms, Math. Ann. 289(1991) 589-612.

[11] G. Shimura, On Eisenstein series, Duke Math. J. 50(1983), 417-476.

Muroran Institute of Technology

27-1 Mizumoto Muroran, 050-8585, Japan

Email:hidenori@mmm.muroran-it.ac.jp 